\title{
Detection of Neurotoxicity Through the Comparison of Jaundiced Infants with and without Blood Transfusion with Normal Infants: An ABR Documentation
}

\author{
Shirin Khan Mohammadi Salehi ${ }^{1}$, Bahram Jalaei ${ }^{2 *}$, Mohsen Ahadi $^{2}$ and Mohammad Maarefvand ${ }^{2}$ \\ ${ }^{1}$ Bachelor degree, Audiologist, Iran \\ ${ }^{2}$ Department of Audiology, Iran University of Medical Sciences, Iran
}

Submission: July 06, 2021; Published: August 16, 2021

*Corresponding author: Bahram Jalaei, Department of Audiology, Faculty of Rehabilitation, Nezam Alley, Shahid Shahnazari St, Mirdamad Blvd, Tehran, Iran

\section{Abstract}

Objective: The main goal of this study is to examine the differences in response with click and chirp stimuli between infants with and without blood exchange transfusions and if there is a significant difference, this could be due to nerve damage, and therefore in these infants, it may also be indicative of future neural damage.

Method: In this study, 59 infants (59 ears) of both sexes aged from 4 to 9 months were included as cases. Of these, 23 were healthy infants (16 boys, 7 girls), 20 were jaundiced (12 boys, 8 girls), and 16 were infants with jaundice and blood exchange transfusions ( 7 boys, 9 girls). Auditory brainstem response test using click $100 \mu \mathrm{sec}$ and chirp stimuli at $60 \mathrm{~dB} \mathrm{nHL}$ was done. Waves I, III, and V were marked using a cursor. The latency of the wave V and I - III, III - V, and I - V latency intervals were recorded.

Results: Findings of the present study showed that the latency times obtained with the chirp stimulus have a shorter latency than the click. Another notable finding in this study was the increasing trend of latency of all waves with chirp stimulus that means in all the waves I, III and V with the use of chirp stimulus, blood transfusion group has the longest latency, followed by the jaundice group and the normal group, respectively.

Conclusion: This finding is highly indicative of neurotoxicity in the blood transfusion group despite the passage of four months after treatment.

Keywords: Hyperbilirubinemia; Jaundiced infants; Neurological lesions; ABR test

\section{Introduction}

One of the most common pathologies in the newborn period is hyperbilirubinemia [1,2]. One study found that this rate is $60 \%[1,3,4]$. The correlation between levels of bilirubin and neurological complications is unclear. While some reports suggest that the nervous system is more affected by increased bilirubin levels, other reports suggest that while a bilirubin level is toxic to one infant it may not be necessarily harmful to another. The reason for this variability is not well known [1].

Many researchers have reported a link between hyperbilirubinemia and damage to the auditory system, while others have not seen any abnormalities [1,5-11]. The nuclei of the auditory brainstem and the inferior colliculus are affected by hyperbilirubinemia. In addition, abnormalities in the neurons of spiral gangli on, auditory fibers [1], Cochlear nuclei [8], superior olive complex, and lateral lemniscus [12], are reported. The fact that the auditory nuclei of the brainstem are the first to be affected by hyperbilirubinemia shows that these effects on the auditory system can be different from their effects on other parts of the nervous system. It can also lead to the hypothesis that lower levels of bilirubin are more likely to damage the auditory system than other parts of the central nervous system because the auditory nuclei of the brainstem are the first to be affected by hyperbilirubinemia [1].

A review of studies shows that Auditory Brainstem Response (ABR) results are abnormal in infants with hyperbilirubinemia [13-16] which usually manifests itself as an increase in latency of the waves $[1,6,8,12,15,17,18]$. In other studies, this abnormality is in the form of an increase in the threshold of wave 
$\mathrm{V}[1,8,17,18]$, increased interpeak latencies [1,12,19-24], loss of one or more waves $[12,18]$ and decrease in amplitudes [6,19]. ABR abnormalities in infants indicated ototoxicity due to high bilirubin $[10,11,15]$. On the other hand, in some studies, researchers have found any abnormalities in the groups of patients $[15,16,25]$. Some researchers have found no link between overall bilirubin levels and threshold and latency changes [18]. The percentage of abnormal ABRs decreases by at least $50 \%$ after treatment $[1,26]$. Only one study found that $100 \%$ of people recovered after 3 months [27]. In a study of $14.3 \%$ of individuals, auditory brainstem response remained abnormal at $14.4 \pm 2.2$ weeks [12].

In 2017, El-Attar et al. reported that Wave V by chirp stimulus has a larger amplitude and lesser latencies in children with moderate to severe sensorineural hearing loss [28]. Maloff and Hood (2014) conducted a study to compare ABRs with both clickand-chirp stimuli in normal adults and adults with sensorineural hearing loss and concluded that ABR with chirp stimulus has a larger peak to peak amplitude and possibly is closer to behavioral thresholds [29]. Higher synchronization of components results in increased response amplitude, thus reducing the time required to perform the test, which is important in neonatal testing [29-35]. While Kristensen and Elberling in 2012 reported that the latency of the wave $V$ with chirp stimulus is greater than the click stimulus in low intensities [32,36]. Other studies also suggest that the wave $\mathrm{V}$ amplitude is larger than click stimulus in the low intensities [32,33,36-43]. Other studies confirm the importance of increasing synchronization, resulting in faster emergence of response and shorter test time in infants $[43,44]$.

Although the value of ABR in the early detection of neuropathy in infants with hyperbilirubinemia over the last 20 years has been reported in articles, its value in predicting neurological disorders is still unclear [22,23,44-46]. Infants with hyperbilirubinemia are at risk for neurological complications. In 2001, Yilmaz and colleagues conducted a study to assess the value of click ABR in predicting the neurological complications of infants with hyperbilirubinemia. They concluded that click ABRs did not provide reliable information on the neurological consequences of hyperbilirubinemia [48].

This study is undertaken to compare the absolute latencies of the peaks I, III and V and I-V, I-III and III-V latency intervals of ABRs with both click and chirp stimulus in infants with blood exchange transfusion (who are likely to develop neurological disorders in the future) and infants without blood exchange transfusions. Thus, this study wants to examine the differences in response between click-and-chirp stimulus in infants with and without blood exchange transfusions and if there is no difference, such as the neuropathy spectrum, this could be due to nerve damage, and therefore in these infants, it may also be indicative of future neural damage.

\section{Material and Methods}

This study is a case-control and double-blind type because the interpretation of the test results is done by two audiologists who are not aware of the status of the samples and on the other hand, the patients participating in the project are not aware of their groups and in which group they are. The study protocols and procedures were approved by the ethics committee of the XX university of Medical Sciences with the code of ethics REC.1397.671. In this study, 59 infants (59 ears) of both sexes aged from 4 to 9 months were included as cases. Of these, 23 were healthy infants ( 16 boys, 7 girls), 20 were jaundiced ( 12 boys, 8 girls), and 16 were infants with jaundice and blood exchange transfusions ( 7 boys, 9 girls) were admitted to the study from April to March 2019.

The classified random sampling method was used so that by using the table of random numbers each of the files was given a number and then randomly selected from the files of infants who have entry conditions. The inclusion criteria were:

A. to be term infant

B. to pass at least 4 months since the treatment of hyperbilirubinemia

C. having ABR with click stimulus

D. lack of middle ear problems and conductive hearing loss

E. lack of problems during pregnancy according to the parents claim (such as hypoxia, hypothermia, etc.)

F. lack of problems during birth according to the parents claim (such as meningitis, cranial facial abnormalities, etc.)

G. lack of pregnancy infections according to the parents claim (such as TORCH (toxoplasmosis, other infections, rubella, cytomegalovirus infection and herpes simplex) and Sepsis, etc.)

H. lack of a family history of hearing loss (including congenital or genetic hearing loss)

I. lack of congenital or metabolic diseases associated with hyperbilirubinemia such as thalassemia, Gilbert's syndrome, galactosemia, defects in the enzyme glucose-6-phosphate dehydrogenase, etc. and exclusion criteria was the unwillingness of the parents to continue their cooperation. The criteria for phototherapy and blood exchange transfusion in infants were based on the protocol of one of the hospitals of the university, which varies according to the amount of bilirubin, duration of infection, weight, etc.

Indication of initiation of phototherapy based on risk areas:

For low-risk infants (more than 38 weeks of gestation without hemolysis and other predisposing factors)

i. For the first 24 hours of birth: based on the opinion of the physician

ii. For 24 to 48 hours of birth: Bilirubin 12milligram(mg)/ deciliter(dL) or more

iii. For 48 to 72 hours of birth: Bilirubin $14 \mathrm{mg} / \mathrm{dL}$ or more

iv. After 72 hours of birth: $16 \mathrm{mg} / \mathrm{dL}$ or more 


\section{Global Journal of Otolaryngology}

Blood exchange indicators based on risk areas:

For low-risk infants (more than 38 weeks of gestation without hemolysis and other predisposing factors)

a. For the first 24 hours of birth: based on the opinion of the physician

b. For the first 24 to 48 hours of birth: bilirubin $18 \mathrm{mg} / \mathrm{dL}$

c. For the first 48 to 72 hours of birth: bilirubin $20 \mathrm{mg} / \mathrm{dL}$

d. After 72 hours of birth: bilirubin 20 to $25 \mathrm{mg} / \mathrm{dL}$

The study protocols and procedures were approved by the ethics committee of the university and written informed consent form of all participants were obtained. After determining the samples and obtaining the consent of the newborn parents to participate in the research, the background information was received and recorded in the test session and before starting the test. The case group was selected from infants with hyperbilirubinemia who were divided into two groups of infants with and without blood exchange transfusions. The Control group was randomly selected from normal infants. Parents were told to wash the baby's forehead and mastoid thoroughly before visiting. Each baby was examined by a pediatrician before the test to determine the dosage of the sleeping drug. First, an otoscopic examination was performed to ensure the health of the external and middle ear. To rule out a conductive lesion in any infant, Immittance acoustic test with the Madsen zodiac 901 model made in Denmark was used while the probe tone frequency was $226 \mathrm{~Hz}$. Normal static compliance and middle ear pressure were $\geq 0.2$ and a range of +50 to -100 , respectively.

Transient Otoacoustic Emission Test (TEOAE) with a vivo sonic device made in Canada were performed. In the TEOAE test, a click stimulus with 80 microseconds duration at $80 \mathrm{~dB}$ sound pressure level (SPL) was used. The results of the TEOAE test were divided into "pass" or "fail" based on the following protocol: To be on the pass group, the SNR had to be equal to 6 decibels in at least three frequency bands of the five frequency bands. ABRs were obtained by vivo sonic response recording device (120-5525 Eglinton Ave. W. Toronto, ON M9C 5K5). The test was performed in a quiet room while the infant was asleep naturally or with the drug (chloral hydrate) in the supine position. The skin surface where the electrode would be applied was first scrubbed with abrasive gel. Disposable electrodes were extracted from their sterile packaging and pressed firmly into place as soon as the plastic protective covering is removed. The electrodes were applied on the head in the following order: Non-inverting electrodes on the mastoid of the test ear and the none test ear, inverted electrode high in the midline of the forehead, and the ground electrode low in the midline of the forehead. The impedance of the electrodes did not exceed $5 \mathrm{k} \Omega$ and inter-electrode impedances were kept under $5 \mathrm{k} \Omega$.

SOAP-Kalman Weighted algorithm was used which provides easier response detection under non-ideal conditions and facilitates non-sedated ABR measurement. The $10 \Omega$ ER3A insert earphone was placed inside the infant's ear. The infant's Electro Encephalon Gram (EEG) range was monitored to minimize noise (lesser than $20 \mu \mathrm{v}$ ). Sampling was discontinued whenever artifacts were large on the monitoring oscilloscope. The order of ear tested was counterbalanced across subjects within each group. Monaural acoustic stimulus, including 100 microseconds click, with rarefaction, condensation, and the alternating polarity was presented. Each of the polarities, which had a better morphology, was selected and the test continued. An intensity of $60 \mathrm{~dB}$ nHL (i.e., relative to the mean threshold in normal hearing subjects) was used. The rate was 11.1 and the filter $30-3000 \mathrm{~Hz}$ was used, meanwhile, the notch filter was on. The time window was 12 milliseconds. For recording each response, 2000 samples were averaged. A one-minute break was considered between each recording. Duplicate recordings were made to check reproducibility.

The presence or absence of Waves I, III, and V were analyzed. Waves I, III, and V were marked using a cursor. The latency of the wave V and I-III, III-V, and I-V latency intervals were recorded. Then the chirp stimulus with the same features $(60 \mathrm{dBnHL}$ intensity, rate 11.1 and 30-3000 Hz filter with notch filter on) was used to record the response. Finally, the answers recorded were interpreted by two skilled audiologists who did not know the condition of the patients and their bilirubin levels. A two-way analysis of variance was used to compare the mean and standard deviation of each ABR variable between different groups of subjects.

\section{Results}

As mentioned earlier, tympanometry and TEOAE tests were performed to rule out the presence of a conductive lesion. According to the results of these tests, presence of conductive lesion in infants' ears was ruled out. The main test in this study is the ABR test, which will be described below. The results of ABR including absolute latencies of the peaks I, III, V and latency intervals (mean \pm SD) of different groups are shown in (Tables $1 \& 2$ ). The mixed ANOVA was performed on the data to answer questions regarding the presence of statistical significance between three groups in terms of a within-group comparison (the type of stimuli i.e., clicks versus chirps). The following paragraphs (1 to 4 ) explain the results of those questions. The latency of wave I $(\mathrm{F}=5.16, \mathrm{df}=45 \mathrm{p}=0.01)$ and wave III $(\mathrm{F}=4.27, \mathrm{df}=47 \mathrm{p}$ $=0.02$ ) were significantly different in the three groups. The post hoc analysis showed that the mean latency of wave I and III in the blood exchange transfusion group were significantly higher than the normal group. Also, the latency of wave I and III in the blood exchange transfusion group were significantly higher than the jaundice group, but the difference between the normal group and the jaundice group were not significant (Figures 1\&2).

The effect of stimulus reached statistical significance. The average latency of the wave $\mathrm{V}$ with click was 6.49 milliseconds and with chirp was 5.83 milliseconds, and the latency of the clicks 
was significantly longer (Figure 3). The standard error was 0.06 for clicks and 0.09 for chirps $(F=4.8, \mathrm{df}=52 \mathrm{p}=0.03) \mathrm{I}-\mathrm{V}$ latency interval with both click and chirp stimuli were significantly different in the three groups. The largest difference between the two groups was jaundice and blood exchange transfusion, so that the I-V latency interval in jaundice was more than blood exchange transfusion (Figure 4). Also, I-V latency intervals in jaundice group was longer than the normal group. The differences between groups and gender and stimuli were not significant. The I-III and III-V latency intervals between groups and gender and stimuli were not significantly different.

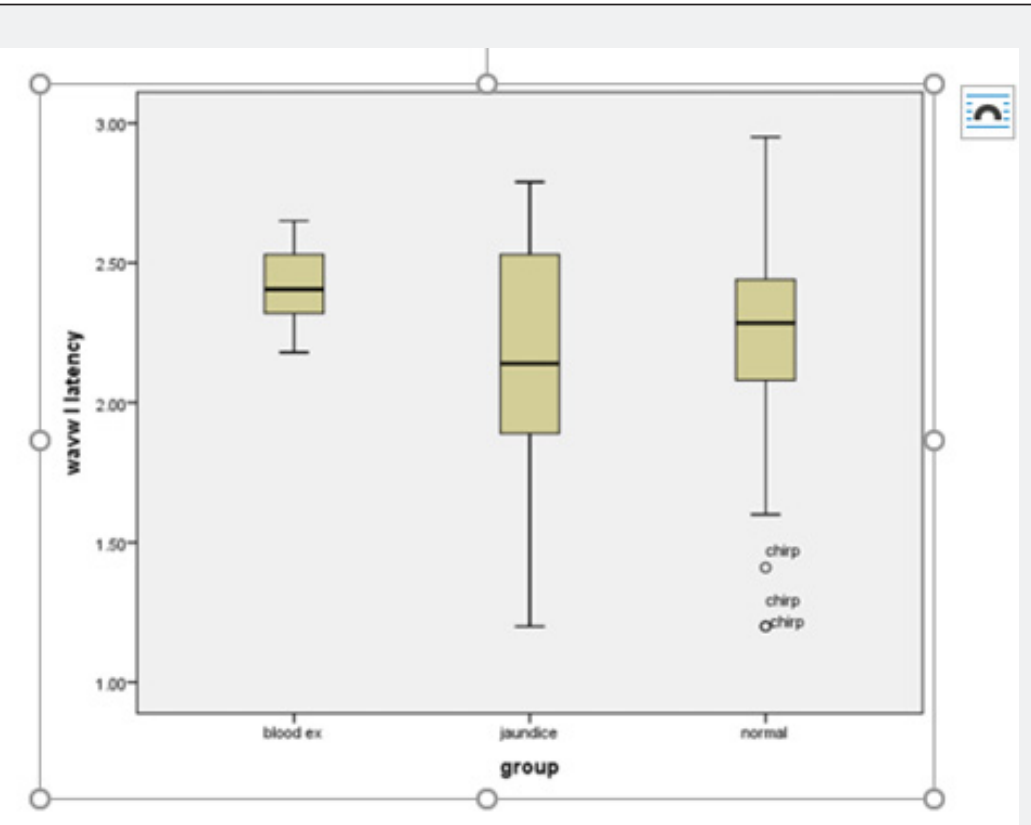

Figure 1: Box plot of wave I latency (bold line across box, median; 25th and 75th centiles; extension, largest and smallest values) in various group of neonates. See text for significance of statistical of comparison between different groups.

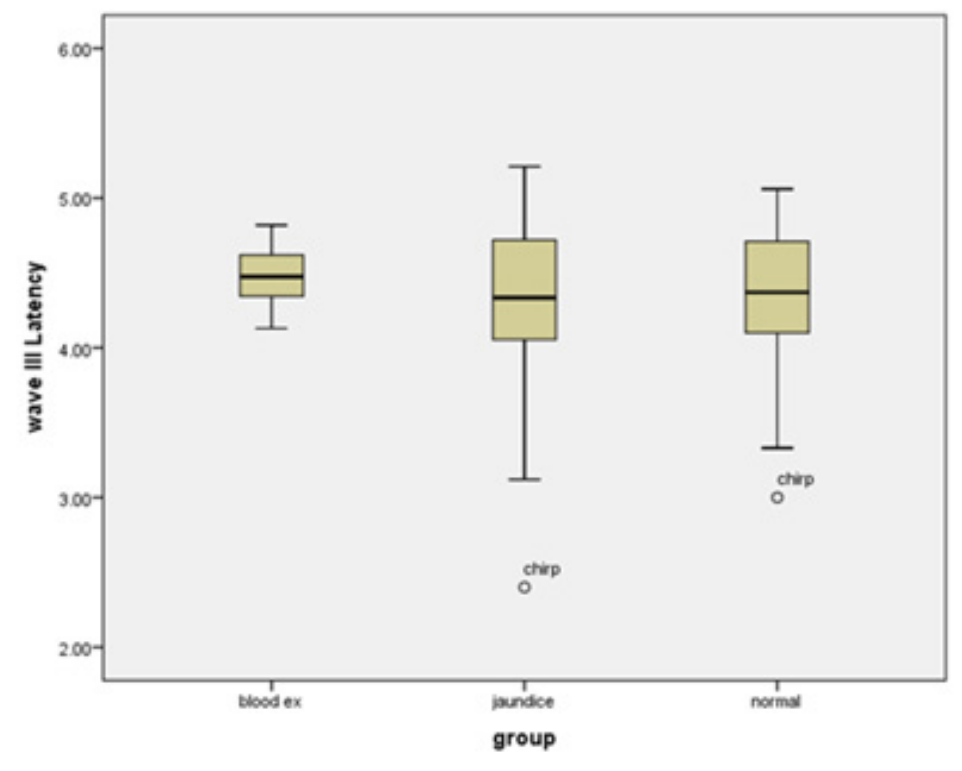

Figure 2: Box plot of wave III latency (bold line across box, median; 25th and 75th centiles; extension, largest and smallest values) in various group of neonates. See text for significance of statistical of comparison between different groups. 


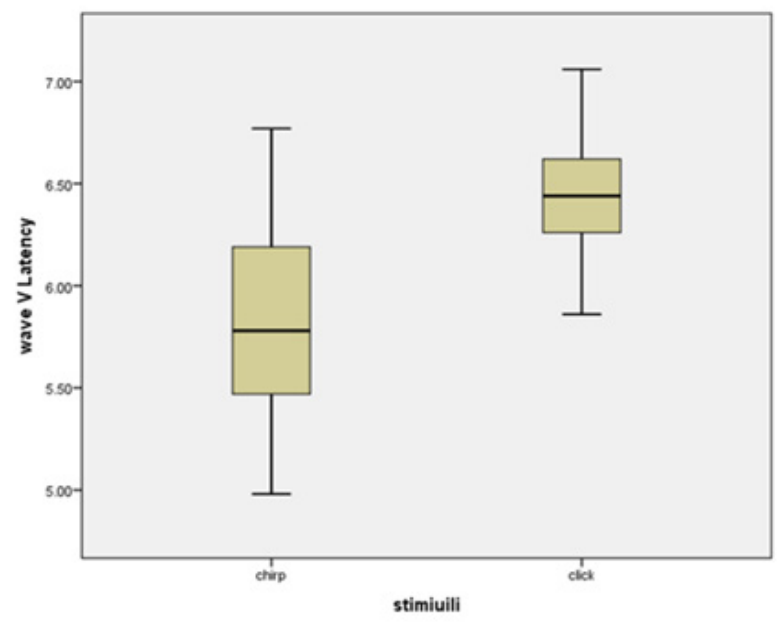

Figure 3: Box plot of wave $V$ latency (bold line across box, median; 25th and 75th centiles; extension, largest and smallest values) in various group of neonates. See text for significance of statistical of comparison between different groups.

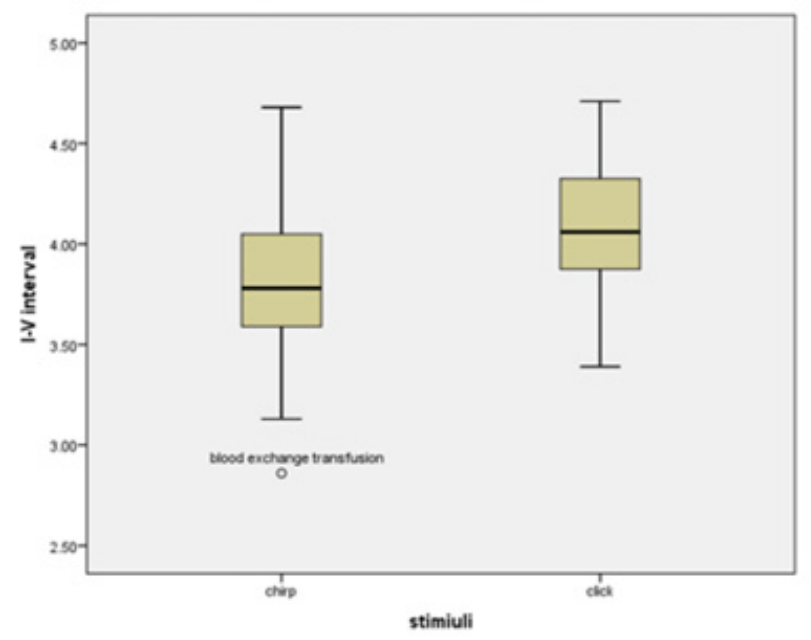

Figure 4: Box plot of I-V latency interval (bold line across box, median; 25th and 75th centiles; extension, largest and smallest values) in various group of neonates. See text for significance of statistical of comparison between different groups.

\section{Discussion}

In this study, brainstem function in processing auditory stimuli using click and chirp stimulation in infants with jaundice and blood exchange transfusion was assessed and compared with normal group infants. By examining the latency of waves, I, III and V, we achieve interesting results. First, in the intragroup comparison, we compared the latency of the obtained waves with the click and chirp stimuli. This study showed the superiority of the chirp stimulus in this comparison, which means that in all the mentioned waves in all three experimental groups, the latency times obtained with the chirp stimulus have a shorter latency than the click. There is only one exception for wave I in the blood transfusion group, where the latency obtained with the click stimulus is 0.03 times shorter than the latency with the chirp stimulus. Overall, this comparison showed that the use of chirp stimuli, even in the jaundice group and the jaundice group with blood transfusion, has reduced the latency of the waves.

In addition, in comparing the latency of waves I, III and $\mathrm{V}$ between groups, we achieved other interesting results that indicated the increasing trend of latency of all waves with chirp stimulus that means in all the mentioned waves with the use of chirp stimulus, blood transfusion group has the longest latency, followed by the jaundice group and the normal group, respectively. This finding is highly indicative of neurotoxicity in 


\section{Global Journal of Otolaryngology}

the blood transfusion group despite the passage of four months after treatment. In the case of click stimulus, only the increasing trend between the three experimental groups for the wave $\mathrm{V}$ is evident, so that the latency of the wave $\mathrm{V}$ is the longest in the blood transfusion group, followed by the jaundice group and the normal group. In the case of waves, I and III, there is no incremental relationship as in wave V. Therefore, in general, it can be concluded that the chirp stimulus had a better performance compared to the click stimulus.

These results are shown in (Table 1) and (Figures 1-3). This finding agreed with studies that reported an increase in the absolute latency of ABR waves in infants with jaundice. Increased latency in infants with jaundice and blood transfusions indicates neurotoxicity of high bilirubin. The analysis of ABR responses showed that the I-III and III-V latency intervals were not significantly different between the groups, but the I-V latency interval in the jaundice group were significantly longer than those of the normal group and blood exchange transfusion. This finding is consistent with studies that have reported increased interpeak intervals in neonates with jaundice.

Table 1: The Mean and standard deviation of wave I, III, V latency for click and chirp stimuli in the three studied groups.

\begin{tabular}{|c|c|c|c|c|c|c|c|}
\hline & & & & \multicolumn{2}{|c|}{ Click Stimulus } & \multicolumn{2}{|c|}{ Chirp Stimulus } \\
\hline & Group & Gender & Number & Mean & Standard deviation & Mean & Standard deviation \\
\hline \multirow{9}{*}{$\begin{array}{l}\text { Wave I } \\
\text { latency }\end{array}$} & \multirow{3}{*}{ Normal } & Male & 7 & 2.32 & 0.17 & 1.88 & 0.48 \\
\hline & & Female & 16 & 2.44 & 0.19 & 1.93 & 0.39 \\
\hline & & total & 23 & 2.4 & 0.19 & 1.91 & 0.41 \\
\hline & \multirow{3}{*}{ Jaundice } & Male & 8 & 2.27 & 0.28 & 2.05 & 0.45 \\
\hline & & Female & 12 & 2.4 & 0.26 & 1.84 & 0.48 \\
\hline & & total & 20 & 2.35 & 0.27 & 1.93 & 0.46 \\
\hline & \multirow{3}{*}{$\begin{array}{c}\text { Blood } \\
\text { exchange } \\
\text { transfusion }\end{array}$} & Male & 9 & 2.35 & 0.11 & 2.43 & 0.15 \\
\hline & & Female & 7 & 2.41 & 0.17 & 2.41 & 0.1 \\
\hline & & total & 16 & 2.39 & 0.14 & 2.42 & 0.13 \\
\hline \multirow{9}{*}{$\begin{array}{l}\text { Wave III } \\
\text { latency }\end{array}$} & \multirow{3}{*}{ Normal } & Male & 7 & 4.49 & 0.25 & 3.91 & 0.37 \\
\hline & & Female & 16 & 4.6 & 0.31 & 4.08 & 0.56 \\
\hline & & total & 23 & 4.57 & 0.29 & 4.01 & 0.5 \\
\hline & \multirow{3}{*}{ Jaundice } & Male & 8 & 4.41 & 0.21 & 3.94 & 0.77 \\
\hline & & Female & 12 & 4.64 & 0.27 & 4.08 & 0.62 \\
\hline & & total & 20 & 4.55 & 0.27 & 4.01 & 0.68 \\
\hline & \multirow{3}{*}{$\begin{array}{c}\text { Blood } \\
\text { exchange } \\
\text { transfusion }\end{array}$} & Male & 9 & 4.55 & 0.15 & 4.45 & 0.15 \\
\hline & & Female & 7 & 4.52 & 0.15 & 4.33 & 0.19 \\
\hline & & total & 16 & 4.54 & 0.15 & 4.4 & 0.17 \\
\hline \multirow{9}{*}{$\begin{array}{l}\text { Wave V } \\
\text { latency }\end{array}$} & \multirow{3}{*}{ Normal } & Male & 7 & 6.3 & 0.2 & 5.7 & 0.47 \\
\hline & & Female & 16 & 6.42 & 0.32 & 5.66 & 0.54 \\
\hline & & total & 23 & 6.39 & 0.29 & 5.68 & 0.5 \\
\hline & \multirow{3}{*}{ Jaundice } & Male & 8 & 6.32 & 0.3 & 5.92 & 0.44 \\
\hline & & Female & 12 & 6.45 & 0.25 & 5.78 & 0.43 \\
\hline & & total & 20 & 6.4 & 0.27 & 5.84 & 0.43 \\
\hline & \multirow{3}{*}{$\begin{array}{c}\text { Blood } \\
\text { exchange } \\
\text { transfusion }\end{array}$} & Male & 9 & 6.58 & 0.13 & 5.98 & 0.38 \\
\hline & & Female & 7 & 6.51 & 0.18 & 6.1 & 0.37 \\
\hline & & total & 16 & 6.55 & 0.15 & 6.03 & 0.37 \\
\hline
\end{tabular}




\section{Global Journal of Otolaryngology}

Table 2: The Mean and standard deviation of I-III, III-V, I-V latency intervals for click and chirp stimuli in the three studied groups.

\begin{tabular}{|c|c|c|c|c|c|c|c|}
\hline & & & & & ck Stimulus & & rp Stimulus \\
\hline & Group & Gender & Number & Mean & Standard deviation & Mean & Standard deviation \\
\hline \multirow{9}{*}{$\begin{array}{l}\text { Wave I } \\
\text { latency }\end{array}$} & \multirow{3}{*}{ Normal } & Male & 7 & 2.32 & 0.17 & 1.88 & 0.48 \\
\hline & & Female & 16 & 2.44 & 0.19 & 1.93 & 0.39 \\
\hline & & total & 23 & 2.4 & 0.19 & 1.91 & 0.41 \\
\hline & \multirow{3}{*}{ Jaundice } & Male & 8 & 2.27 & 0.28 & 2.05 & 0.45 \\
\hline & & Female & 12 & 2.4 & 0.26 & 1.84 & 0.48 \\
\hline & & total & 20 & 2.35 & 0.27 & 1.93 & 0.46 \\
\hline & \multirow{3}{*}{$\begin{array}{l}\text { Blood exchange } \\
\text { transfusion }\end{array}$} & Male & 9 & 2.35 & 0.11 & 2.43 & 0.15 \\
\hline & & Female & 7 & 2.41 & 0.17 & 2.41 & 0.1 \\
\hline & & total & 16 & 2.39 & 0.14 & 2.42 & 0.13 \\
\hline \multirow{9}{*}{$\begin{array}{l}\text { Wave III } \\
\text { latency }\end{array}$} & \multirow{3}{*}{ Normal } & Male & 7 & 4.49 & 0.25 & 3.91 & 0.37 \\
\hline & & Female & 16 & 4.6 & 0.31 & 4.08 & 0.56 \\
\hline & & total & 23 & 4.57 & 0.29 & 4.01 & 0.5 \\
\hline & \multirow{3}{*}{ Jaundice } & Male & 8 & 4.41 & 0.21 & 3.94 & 0.77 \\
\hline & & Female & 12 & 4.64 & 0.27 & 4.08 & 0.62 \\
\hline & & total & 20 & 4.55 & 0.27 & 4.01 & 0.68 \\
\hline & \multirow{3}{*}{$\begin{array}{l}\text { Blood exchange } \\
\text { transfusion }\end{array}$} & Male & 9 & 4.55 & 0.15 & 4.45 & 0.15 \\
\hline & & Female & 7 & 4.52 & 0.15 & 4.33 & 0.19 \\
\hline & & total & 16 & 4.54 & 0.15 & 4.4 & 0.17 \\
\hline \multirow{9}{*}{$\begin{array}{l}\text { Wave V } \\
\text { latency }\end{array}$} & \multirow{3}{*}{ Normal } & Male & 7 & 6.3 & 0.2 & 5.7 & 0.47 \\
\hline & & Female & 16 & 6.42 & 0.32 & 5.66 & 0.54 \\
\hline & & total & 23 & 6.39 & 0.29 & 5.68 & 0.5 \\
\hline & \multirow{3}{*}{ Jaundice } & Male & 8 & 6.32 & 0.3 & 5.92 & 0.44 \\
\hline & & Female & 12 & 6.45 & 0.25 & 5.78 & 0.43 \\
\hline & & total & 20 & 6.4 & 0.27 & 5.84 & 0.43 \\
\hline & \multirow{3}{*}{$\begin{array}{l}\text { Blood exchange } \\
\text { transfusion }\end{array}$} & Male & 9 & 6.58 & 0.13 & 5.98 & 0.38 \\
\hline & & Female & 7 & 6.51 & 0.18 & 6.1 & 0.37 \\
\hline & & total & 16 & 6.55 & 0.15 & 6.03 & 0.37 \\
\hline
\end{tabular}

\section{Conclusion}

Findings of the present study showed the superiority of the chirp stimulus, which means that in all the mentioned waves in all three experimental groups, the latency times obtained with the chirp stimulus have a shorter latency than the click. Another notable finding in this study was the increasing trend of latency of all waves with chirp stimulus that means in all the waves I, III and $\mathrm{V}$ with the use of chirp stimulus, blood transfusion group has the longest latency, followed by the jaundice group and the normal group, respectively. This finding is highly indicative of neurotoxicity in the blood transfusion group despite passing four months after treatment. Therefore, chirp stimulation seems to be more efficient to click to achieve infantile hearing threshold. But to check the $A B R$ response at high intensities the morphology of the waves was sharper using the click stimulus rather than chirp. Based on the above results, it was concluded that there is a difference between the ABR responses of infants with blood exchange transfusion using chirp stimulation, so the proposed hypothesis that it would predict neurological lesions in these infants was accepted.

\section{References}

1. Akinpelu OV, Waissbluth S, Daniel SJ (2013) Auditory risk of hyperbilirubinemia in term newborns: a systematic review. Int J Pediatr Otorhinolaryngol 77(6): 898-905.

2. Hosono S, Ohno T, Kimoto H, Nagoshi R, Shimizu M, et al. (2002) Follow-up study of auditory brainstem responses in infants with high unbound bilirubin levels treated with albumin infusion therapy. Pediatr Int 44(5): 488-492. 


\section{Global Journal of Otolaryngology}

3. Shapiro SM (2003) Bilirubin toxicity in the developing nervous system. Pediatr Neurol 29(5): 410-421.

4. Sheykholeslami K, Kaga K (2000) Otoacoustic emissions and auditory brainstem responses after neonatal hyperbilirubinemia. Int J Pediatr Otorhinolaryngol 52(1): 65-73.

5. Hall III J (2015) eHandbook of auditory evoked responses: principles, procedures \& protocols. Pretoria: Pearson.

6. Amin SB, Ahlfors C, Orlando MS, Dalzell LE, Merle KS, et al. (2001) Bilirubin and serial auditory brainstem responses in premature infants. Pediatrics 107(4): 664-670.

7. Ogun B, Şerbetçioğlu B, Duman N, Özkan H, Kırkım G (2003) Long-term outcome of neonatal hyperbilirubinaemia: subjective and objective audiological measures. Clin Otolaryngol Allied Sci 28(6): 507-513.

8. Rhee CK, Park HM, Jang YJ (1999) Audiologic evaluation of neonates with severe hyperbilirubinemia using transiently evoked otoacoustic emissions and auditory brainstem responses. Laryngoscope 109(12): 2005-2008

9. Sano M, Kaga K, Kitazumi E, Kodama K (2005) Sensorineural hearing loss in patients with cerebral palsy after asphyxia and hyperbilirubinemia. Int J Pediatr Otorhinolaryngol 69(9): 1211-1217.

10. Soares I, Collet L, Delorme C, Salle B, Morgon A (1989) Are click-evoked BAEPs useful in case of neonate hyperbilirubinemia? Int J Pediatr Otorhinolaryngol 17(3): 231-237.

11. Streletz L, Graziani L, Branca P, Desai H, Travis S, et al. (1986) Brainstem auditory evoked potentials in full term and preterm newborns with hyperbilirubinemia and hypoxemia. Neuropediatrics 17(2): 66-71.

12. Agrawal V, Shukla R, Misra P, Kapoor R, Malik G (1998) Brainstem auditory evoked response in newborns with hyperbilirubinemia. Indian Pediatr 35(6): 513-518.

13. Ahlfors CE, Parker AE (2008) Unbound bilirubin concentration is associated with abnormal automated auditory brainstem response for jaundiced newborns. Pediatrics 121(5): 976-978.

14. Ip S, Lau J, Chung M, Kulig J, Sege R, et al. (2004) Hyperbilirubinemia and kernicterus: 50 years later. Pediatrics 114(1): 263-264.

15. Jiang ZD, Chen C, Liu TT, Wilkinson AR (2007) Changes in brainstem auditory evoked response latencies in term neonates with hyperbilirubinemia. Pediatr Neurol 37(1): 35-41.

16. Jiang ZD, Wilkinson AR (2014) Impaired function of the auditory brainstem in term neonates with hyperbilirubinemia. Brain Dev 36(3): 212-218.

17. Bhagya V, Brid S, Vinod A, Dhawal S (2014) Evaluation of auditory \& brainstem responses in hyperbilirubinemic infants. J Evol Med Dent Sci 3(31): 8579-8585.

18. Silva DPCd, Martins RHG (2009) Analysis of transient otoacoustic emissions and brainstem evoked auditory potentials in neonates with hyperbilirubinemia. Braz J Otorhinolaryngol 75(3): 381-386.

19. Shapiro SM, Popelka GR (2011) Auditory impairment in infants at risk for bilirubin-induced neurologic dysfunction. Seminars in perinatology, Elsevier.

20. Chisin R, Perlman M, Sohmer H (1979) Cochlear and brain stem responses in hearing loss following neonatal hyperbilirubinemia. Ann Otol Rhinol Laryngol 88(3): 352-357.

21. Lenhardt M, McArtor R, Bryant B (1984) Effects of neonatal hyperbilirubinemia on the brainstem electric response. J pediatrics 104(2): 281-284

22. Nakamura H, Takada S, Shimabuku R, Matsuo M, Matsuo T, et al. (1985) Auditory nerve and brainstem responses in newborn infants with hyperbilirubinemia. Pediatrics 75(4): 703-708.
23. Perlman M, Fainmesser P, Sohmer H, Tamari H, Wax Y, et al. (1983) Auditory nerve-brainstem evoked responses in hyperbilirubinemic neonates. Pediatrics 72(5): 658-664.

24. Kotagal S, Rudd D, Rosenberg C, Horenstein S (1981) Brainstem Auditory Evoked Potentials in Neonatal Hyperbilirubinemia: PP 21 Neurology 31(4).

25. Thoma J, Gerull G, Mrowinski D (1986) A long-term study of hearing in children following neonatal hyperbilirubinemia. Arch Otorhinolaryngol 243(2): 133-137.

26. Wennberg RP, Ahlfors CE, Bickers R, McMurtry CA, Shetter JL (1982) Abnormal auditory brainstem response in a newborn infant with hyperbilirubinemia: improvement with exchange transfusion. J Pediatr 100(4): 624-626.

27. Gupta A, Raj H, Anand N (1990) Auditory brainstem responses (ABR) in neonates with hyperbilirubinemia. Indian J Pediatr 57(5): 705-711.

28. El Attar AM, Enass SM, Abu MMH, Sanaa MM (2017) Comparison of chirp and click-evoked brainstem response stimulus in children with moderate and severe sensorineural hearing loss. J Curr Med Res Pract 2(2): 99-104.

29. Maloff ES, Hood LJ (2014) A comparison of auditory brain stem responses elicited by click and chirp stimuli in adults with normal hearing and sensory hearing loss. Ear Hear 35(2): 271-282.

30. Keesling DA, Parker JP, Sanchez JT (2017) A comparison of commercially available auditory brainstem response stimuli at a neurodiagnostic intensity level. Audiol Res 7(1): 15-22.

31. Kristensen SG, Elberling C (2012) Auditory brainstem responses to level-specific chirps in normal-hearing adults. J Am Acad Audiol 23(9): 712-721.

32. Cobb KM, Stuart A (2016) Neonate auditory brainstem responses to CE-chirp and CE-chirp octave band stimuli I: versus click and tone burst stimuli. Ear Hear 37(6): 710-723.

33. Mühler R, Rahne T, Verhey JL (2013) Auditory brainstem responses to broad-band chirps: Amplitude growth functions in sedated and anaesthetised infants. Int J Pediatr Otorhinolaryngol 77(1): 49-53.

34. Xu Zm, Cheng Wx, Yao Zh (2014) Prediction of frequency-specific hearing threshold using chirp auditory brainstem response in infants with hearing losses. Int J Pediatr Otorhinolaryngol 78(5): 812-816.

35. Ferm I, Lightfoot G (2015) Further comparisons of ABR response amplitudes, test time, and estimation of hearing threshold using frequency-specific chirp and tone pip stimuli in newborns: Findings at 0.5 and $2 \mathrm{kHz}$. Int J Audiol 54(10): 745-750.

36. Sabet VK, Mahdavi Zafarghandi ME, Safavi M, Sharifian M, Tabatabaee SM (2014) Comparison of click and CE-chirp-evoked human auditory brainstem responses: a preliminary study. Audiol 23(4): 69-76.

37. Cebulla M, Hofmann S, Shehata Dieler W (2014) Sensitivity of ABR based newborn screening with the MB11 BERAphone ${ }^{\circledR}$. Int J Pediatr Otorhinolaryngol 78(5): 756-761.

38. Dau T, Wegner O, Mellert V, Kollmeier B (2000) Auditory brainstem responses with optimized chirp signals compensating basilarmembrane dispersion. J Acoust Soc Am 107(3): 1530-1540.

39. Petoe MA, Bradley AP, Wilson WJ (2010) On chirp stimuli and neural synchrony in the suprathreshold auditory brainstem response. J Acoust Soc Am 128(1): 235-246.

40. Elberling C, Kristensen SG, Don M (2012) Auditory brainstem responses to chirps delivered by different insert earphones. J Acoust Soc Am 131(3): 2091-2100

41. Fobel 0, Dau T (2004) Searching for the optimal stimulus eliciting auditory brainstem responses in humans. J Acoust Soc Am 116(4): 2213-2222. 
42. Bargen GA (2015) Chirp-evoked auditory brainstem response in children: a review. Am J Audiol 24(4): 573-583.

43. Elberling C, Don M (2010) A direct approach for the design of chirp stimuli used for the recording of auditory brainstem responses. J Acoust Soc Am 128(5): 2955-2964.

44. Vohr BR (1990) New approaches to assessing the risks of hyperbilirubinemia. Clin Perinatol 17(2): 293-306.

45. Stein L, Tremblay K, Pasternak J, Banerjee S, Lindemann K, et al. (1996) Brainstem abnormalities in neonates with normal otoacoustic emissions. Seminars in Hearing, Thieme Medical Publishers, Inc, US.
46. Duara S, Suter CM, Bessard KK, Gutberlet RL (1986) Neonatal screening with auditory brainstem responses: results of follow-up audiometry and risk factor evaluation. J Pediatr 108(2): 276-281.

47. Yilmaz Y, Degirmenci S, Akdas F, Külekçi S, Çiprut A, et al. (2001) Prognostic value of auditory brainstem response for neurologic outcome in patients with neonatal indirect hyperbilirubinemia. J Child Neurol 16(10): 772-775. 